\title{
Promoting a Research-Based Education through Undergraduate Research Experience for Engineering Students
}

\author{
${ }^{* 1}$ Maya Abi Akl, ${ }^{1}$ Othmane Bouhali, and ${ }^{1}$ Yassine Toufique \\ ${ }^{1}$ Science Program, Texas A\&M University at Qatar, Doha, Qatar
}

\begin{abstract}
Various studies have shown the crucial and strong impact that undergraduate research has on the learning outcome of students and its role in clarifying their career path. Therefore, many colleges and universities are promoting undergraduate research experience amongst their students. Texas A\&M University at Qatar (TAMUQ), a branch campus of Texas A\&M University in College Station in the state of Texas and one of the six American University campuses in Education City, Qatar is actively involving its engineering students in research projects spanning different disciplines across its academic programs. This paper describes how the High Energy and Medical Physics Group at TAMUQ supports and engages the undergraduate students in research activities, summarizes the outcomes of their work and the impact on their career.
\end{abstract}

Key words: Undergraduate Research, education, engineering, simulation

\section{Introduction}

Promoting research at the undergraduate level is essential to build an enriched learning environment for students [1,2]. They will get exposed to the research world at an early stage, acquire new skills, learn about fundamental topics in depth, develop a professional identity and have a clearer idea about the career path they want to follow later. Undergraduate research fosters engaged learning and therefore is considered as a high-impact educational practice that must be implemented to achieve excellence.

The High Energy and Medical Physics Group at TAMUQ has been supporting and engaging undergraduate students in different research projects in the areas of High Energy Physics and Medical Physics for the past six years. It attracted students from all four majors offered at TAMUQ, Electrical, Mechanical, Chemical and Petroleum Engineering shortly after the launching of its research activities. Many projects conducted within the group were awarded by the Qatar National Research Fund which is a governmental funding body that provides funding to highly competitive projects that address national priorities and contribute to capacity building [3]. Students were trained to use a high performance computing facility, different programming languages, software and Monte Carlo based platforms for their simulation. As for the outcomes, they participated and presented at international conferences, many of them attended CERN [4] summer internship program and took part in different hands-on activities within the Compact Muon Solenoid (CMS) experiment [5] at CERN. Some of the results produced by students were even published in

*Corresponding author: Address: Texas A\&M University at Qatar, Education City, P.O. Box 23874, Doha, Qatar. Email address: maya.abi_akl@qatar.tamu.edu, Phone: +974-44230202 
scientific journals.

In this paper, we highlight some of the various projects that our students completed, the different tools that were used, as well as the research outcomes. Then, we will discuss the impact of this experience on their learning and undergraduate education as well as their career path, especially their postgraduate studies.

\section{Selected Projects}

The completed and ongoing projects fall into two main categories; High Energy Physics and Medical Physics. Some of them were awarded by QNRF which covers travel expenses for students as well as mentors to participate in conferences, cost of purchasing equipment or software needed to conduct the research in addition to a yearly stipend for students.

While other projects were conducted without any funding, solely driven by the motivation of the mentors to support students' education and stimulate their interest in the research world. In return the students showed commitment to learning and contributing to new findings in the field.

Throughout all this, TAMUQ provided full support of those research activities by every available mean.

A detailed description of the projects is provided below.

\subsection{Simulation studies and data analysis of charged particle detectors}

The High Energy and Medical Physics group conducted several projects that mainly consisted of the simulation of charged particle detectors like those based on the Gas Electron Multiplier used in the CMS experiment at CERN. The objective was to study their performance as well as perform analysis of data collected in the CMS experiment for the upgrade of their detectors.

For this purpose, the students were trained to access the high performance computing facility at TAMUQ [6], and to use different programming languages like object oriented $\mathrm{C}++$. They also followed extensive training on the specific software needed in their research work, namely ANSYS [7], a computational fluid dynamics package to numerically compute the 3D electromagnetic field map inside the detector, and Garfield ${ }^{++}$, a toolkit for the simulation of particle detectors [8]. Moreover, they were introduced to statistical methods and trained to use it especially in the data analysis part of the projects.

In addition to the aforementioned trainings, weekly meetings were held to follow up on the work, discuss the progress and answer the students' questions. 


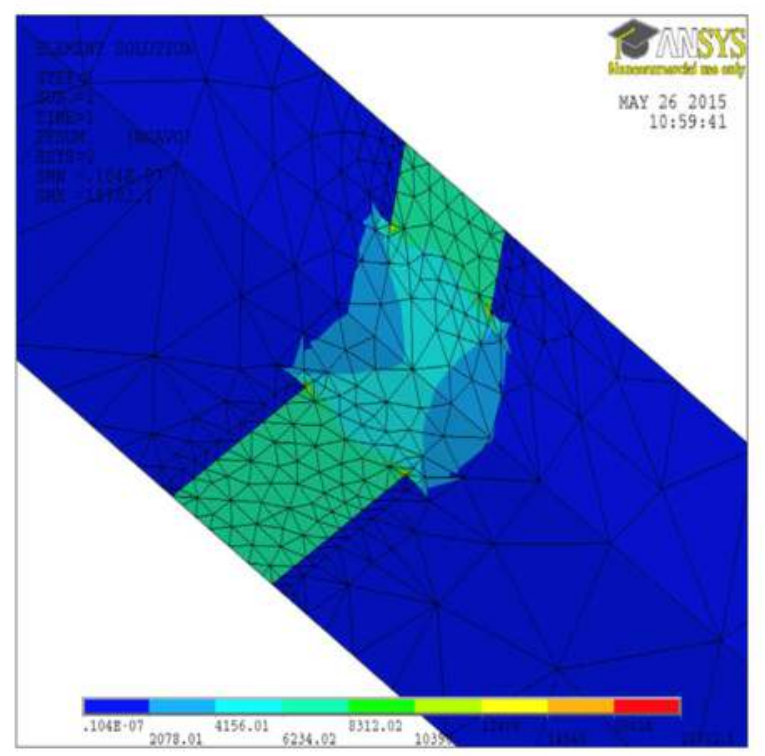

Figure 1. View of the electromagnetic potential tetrahedral mesh as generated by ANSYS [9].

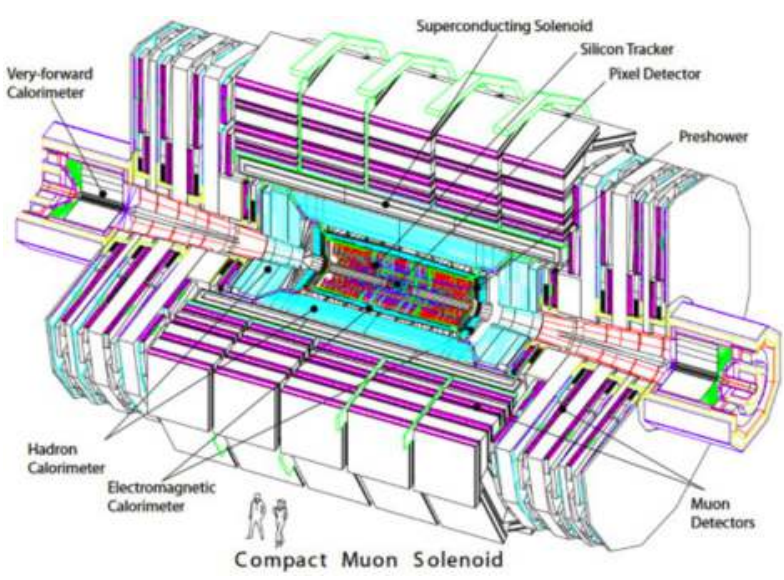

Figure 2. Schematic view of the CMS detector

\subsection{Monte Carlo study of a clinical Positron Emission Tomography scanner}

This work was aimed towards the modeling and validation of Positron Emission Tomography (PET) scanners. PET is a nuclear imaging modality used for both diagnostic and treatment purposes. Again, students were trained to use the high performance computing facility at TAMUQ, $\mathrm{C}++$ programming language as well as GATE, a simulation toolkit adapted to the field of nuclear medicine [10]. The simulation consists of building a model of the scanner given the specifications from the manufacturer and obtaining the performance metrics of the modeled scanner in order to compare to the published measurements. Statistical analysis was also used to analyze output data of the simulation.

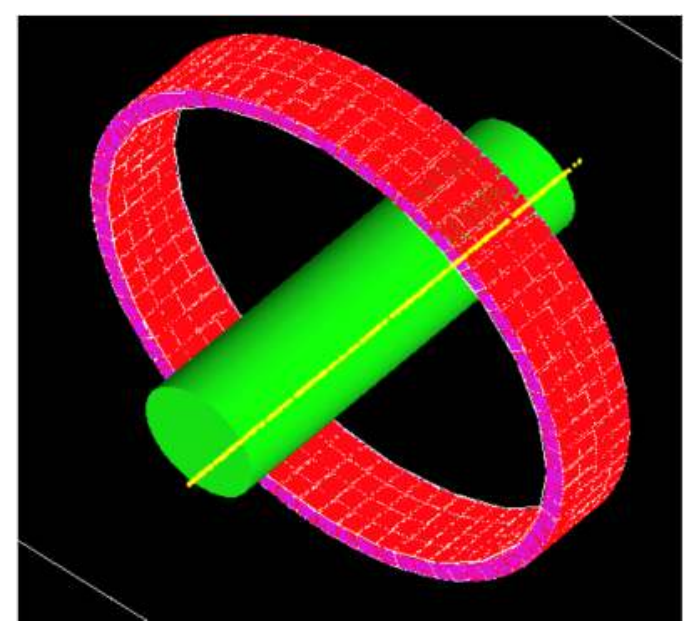

Figure 3. GATE geometry model of a clinical PET scanner with the phantom and the line source [11]. 


\subsection{A framework for solving large scale optimization models under uncertainty for cancer treatment using radiation therapy}

The goal of this ongoing project is to establish a fast and individualized radiation therapy planning framework for cancer patients to overcome the limitations of the current treatment planning techniques. GATE software will be used on the high performance computer to model and validate a linear accelerator that will produce the X-ray beam used in Radiotherapy (RT) treatment. Then, the dose distribution profile will be calculated and compared to experimental measurements.

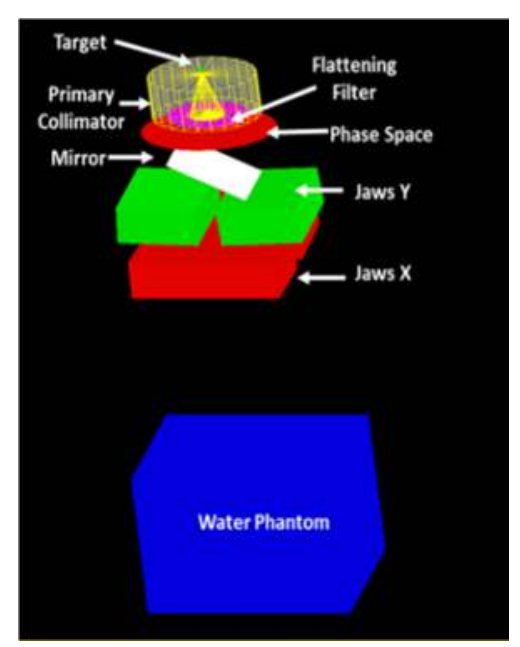

Figure 4. GATE geometry model of the various components of a linear accelerator [12].
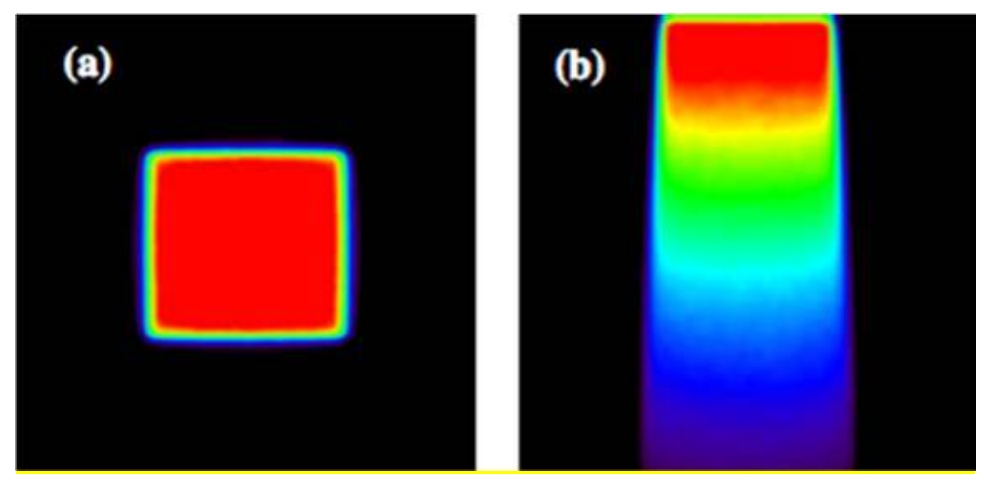

Figure 5. 3D dose distribution in a water phantom. (a) Transverse mode. (b) Coronal mode [12].

Students will be trained to use the knowledge and tools needed to perform the simulation. They will also have the opportunity to gain hands-on experience and work with medical physicists and engineers in hospitals.

\section{Research Outcomes and Impact on career path}

The aforementioned projects were carefully prepared and designed to address the need to introduce innovative and effective strategies to enhance students' learning by extending it outside the classroom. The undergraduate research opportunity that was provided to the engineering students at TAMUQ through these projects was strictly beneficial and quite enlightening to many of them and a turning point to others. Below is a summary list of the main research outcomes of this work:

- Expanding knowledge in courses that they use in their respective engineering curriculum such as statistical analysis and programming.

- Learning to access and use a high performance computing facility, in order to speed up the simulation involving heavy computation. This was also useful for some of their engineering projects.

- Understanding and learning to run different simulation software, out of which some are used in the courses offered in specific engineering disciplines such as ANSYS which is heavily 
used in Mechanical Engineering.

- Publishing papers in scientific journals and conference proceedings $[9,13]$.

- Presenting (oral/poster) at international conferences [14,15].

- Interning/training at CERN and getting exposed to lab work with Scientists and Engineers.

- Learning to work independently as well as in a team and to be part of a large collaboration such as CMS at CERN.

- Training in Medical Imaging and Radiotherapy departments at hospitals.

As for the impact on their career path, many students were positively influenced by the undergraduate research experience, and decided later to pursue a career in research in various prestigious universities such as Stanford University, Columbia University and University College London. Moreover, according to them, the choice of their postgraduate studies program was made easier after their involvement in research activities at an early stage of their education.

\section{Conclusion}

This work shows that engaging engineering students in research activities at the undergraduate level greatly improves their learning curve, allows them to develop professionally and personally and encourages them towards pursuing a research career. Therefore, all universities should promote and support undergraduate research in order to achieve educational excellence. However, the research projects should be carefully designed to address the needs of the students depending on their academic majors.

\section{Acknowledgements}

The authors wish to thank the CMS GEM collaboration, the Qatar National Research Fund as well as the research computing team at TAMUQ for supporting those projects. Moreover, this work wouldn't have been possible without the effort and commitment of the students.

\section{References}

[1] Desai KV, Gatson SN, Stiles TW, Stewart RH, Laine GA, Quick CM. Integrating research and education at research-extensive universities with research-intensive communities. Adv Physiol Educ. 2008;32(2):136-41.

[2] Heidi A. Wayment, K. Laurie Dickson. Increasing student participation in undergraduate research benefits students, faculty, and department. Teaching of Psychology 2008;35:19497.

[3] The Qatar National Research Fund, http://www.qnrf.org

[4] CERN, http://www.cern.ch

[5] The CMS experiment, http://cms.web.cern.ch

[6] TAMUQ Research Computing, http://rc.qatar.tamu.edu

[7] ANSYS software, www.ansys.com

[8] R. Veenhof, Garfield: A drift chamber simulation program. Nucl. Instr. Meth. A 1998;419:726-30.

[9] M. Abi Akl, O. Bouhali, A. Castaneda, Y. Maghrbi, T. Mohamed. Uniformity studies in large 
area triple-GEM based detectors Nucl. Instr. Meth. A 2016;832:1-7.

[10] S. Jan, G. Santin, D. Strul, S. Staelens, K. Assié, D. Autret et al. GATE: a simulation toolkit for PET and SPECT. Phys. Med. Biol. 2004;49:4543.

[11] Rahal Saaidi, Yassine Toufique, Abdelkrim Zeghari, Abderrahman El Kharrim, Rajaâ Cherkaoui El Moursli. GATE simulation of a clinical PET scanner: Influence of windows timing coincidences and dead time on count rate performance. $4^{\text {th }}$ International Conference on Automation, Control Engineering and Computer Science (ACECS 2017). Vol 19, pp.3135.

[12] Zakaria Aitelcadi, Yassine Toufique, Abderrahman El Kharrim, Saad Elmadani, Abderraouf Hilali, Othmane Bouhali. Validation of the GATE Monte Carlo code for radiation: Varian Clinac2300C/D. $4^{\text {th }}$ International Conference on Optimization and Applications, ICOA 2018.

[13] O. Bouhali, Al. Shehryar and T. Mohamed. Accelerating avalanche simulation in gas based charged particle detectors. Nucl. Instr. Meth. A 2018;901:92-8.

[14] T. Mohamed, N. Renganathan, M. Abi Akl, O. Bouhali, Y. Maghrbi, A. Castaneda. Simulation studies of charged particle detectors for collider experiments. Poster presentation at the Annual Research Conference (ARC16), Qatar Foundation, Doha, Qatar.

[15] Abdulla Al-Suwaidi. Investigation of the performances of the Gas Electron Multiplier for collider experiments. Oral presentation at the ARC16, Doha, Qatar. 